\title{
ARTROPLASTIA TOTAL DE QUADRIL COM PRÓTESE BIOMEC: 20 ANOS DE SEGUIMENTO
}

\author{
TOTAL HIP ARTHROPLASTY WITH BIOMEC: 20-YEAR FOLLOW-UP
}

Rodrigo Benedet Scheidt', Ricardo Rosito², Carlos Alberto de Souza Macedo ${ }^{3}$, Carlos Roberto Gália ${ }^{4}$

\section{RESUMO}

Objetivo: Avaliar o desfecho clínico e radiográfico da artroplastia total de quadril (ATQ) com prótese Biomec em um período de 20 anos (1988 a 2008). Métodos: Os pacientes foram avaliados clinicamente segundo a classificação funcional de Merle d'Aubigné e Postel e submetidos à radiografia de bacia em anteroposterior (AP) e da articulação operada em perfil. As variáveis avaliadas foram osteólise acetabular e femoral, desgaste do polietileno, migração da haste, seguimento e desfecho clínico. Resultados: O seguimento médio foi de 10,6 anos (4 a 20 anos); a amostra foi de 84 pacientes, sendo 32 do sexo masculino e 52, do feminino; a idade média no pré-operatório foi de 47 anos; obtivemos 88\% de resultados bons e muito bons na avaliação clínica. Com relação aos achados radiográficos obtivemos 92,5\% de bons resultados. O desgaste médio do polietileno foi de 1,4mm. Conclusão: A utilização da prótese Biomec nesta coorte apresentou bons resultados clínicos e radiográficos.

Descritores - Quadril/cirurgia; Artroplastia de quadril; Biomecânica

\section{INTRODUÇÃO}

A popularização da artroplastia total de quadril (ATQ) foi conduzida por Chanrley na década de $60^{(1)}$. Nessa época a articulação metal sobre polietileno fixada por cimento cirúrgico (polimetilmetacrilato-PMMA) tornouse padrão. Com a ampla difusão da ATQ cimentada aumentaram também as complicações, principalmente o afrouxamento asséptico e as osteólises. Inicialmente, acreditava-se que o problema estava no cimento, época em que o termo “doença do cimento" passou a ser

\section{ABSTRACT}

Objective: To analyze the clinical and radiological outcomes of total hip arthroplasty with Biomec prosthesis over a 20-year period (1988 to 2009). Methods: The patients were evaluated clinically according to the Merle d'Aubigné and Postel functional classification, and submitted to anteroposterior (AP) radiography of the spleen and operated joint in profile. The variables evaluated were acetabular and femoral osteopenia, polyethylene wear, stem migration, follow-up and clinical outcomes. Results: The mean follow-up was 10.6 years (4 to 21years), the sample consisted of 84 patients; 32 male and 52 female; the mean preoperative age was 47 years; we obtained $88 \%$ good or excellent results in the clinical evaluation. In relation to the radiological findings, we obtained $92.5 \%$ good results. The mean polyethylene wear was $1.4 \mathrm{~mm}$. Conclusion: The use of the Biomec prosthesis in this cohort showed good clinical and radiographic results.

Keywords - Hip/surgery; Arthroplasty, Replacement, Hip; Biomechanics

investigado $^{(2)}$. Os resultados desses estudos mostraram que a razão das falhas não estava apenas no cimento, e sim em um conjunto de fatores. De qualquer forma, esses eventos estimularam a busca de soluções biológicas (sem cimento) como alternativas na $\mathrm{ATQ}^{(3,4)}$.

Assim, ao final da década de 70, aumentaram as indicações de ATQ com fixação biológica. Esse período foi marcado pelo surgimento de uma série de novos implantes com concepções bastante variadas quanto ao material, formato, tipo de revestimento e fixação ${ }^{(5)}$. Dentre estes, a prótese de Spotorno ${ }^{\circledR}$, consagrada mun-

1 - Médico Ortopedista R4 do Grupo de Cirurgia do Quadril do Serviço de Ortopedia do Hospital de Clínicas de Porto Alegre (HCPA), RS.

2 - Professor Substituto do Departamento de Cirurgia da UFRGS, Médico Contratado do HCPA e Preceptor do Grupo de Cirurgia do Quadril do HCPA.

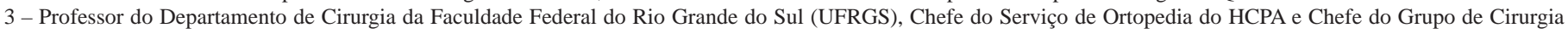
do Quadril do HCPA.

4 - Professor do Programa de Pós-Graduação em Cirurgia da UFRGS, Médico Contratado e Preceptor do Grupo de Cirurgia do Quadril do HCPA.

Trabalho realizado no Departamento de Cirurgia, Ortopedia, Universidade Federal do Rio Grande do Sul - UFRGS - Porto Alegre, RS.

Correspondência: Ricardo Rosito - Rua Ramiro Barcelos, 2.350 - Largo Eduardo Faracco - 90035-903 - Porto Alegre, RS, Brasil. - E-mail: ricardo@rosito.com.br 
dialmente, é um exemplo de implante com fixação biológica, com formato quadrangular cuneiforme de titânio (T16Al7Nb), que serviu de modelo para o desenvolvimento da prótese em estudo (Biomec) ${ }^{(6)}$. A análise dos resultados preliminares de diversos designs de implantes não cimentados, quando comparados aos resultados alcançados pelas próteses adequadamente cimentadas, sugeriram que nem todas as concepções teriam sustentação com o seguimento em médio prazo, fato este bem demonstrado pela variação de modelos feitos por diversas empresas conceituadas na época ${ }^{(7,8)}$.

Tudo isso nos motivou a mostrar nossa experiência de 20 anos em ATQ com a prótese quadrangular e cuneiforme não cimentada de Biomec.

O objetivo deste trabalho é mostrar os resultados clínicos e radiográficos com o uso da prótese não cimentada Biomec com 10,6 anos de seguimento médio.

\section{MÉTODOS}

O presente estudo consiste em coorte de 84 pacientes (93 quadris) submetidos à artroplastia total de quadril com uso da prótese não cimentada Biomec, no período de 1988 a 2005, com seguimento até o ano de 2008. A avaliação clínica foi realizada antes da avaliação radiográfica, além de ter sido feita por examinadores distintos, com o objetivo de diminuir os vícios de aferição. Os pacientes foram chamados para avaliação, responderam a um protocolo preestabelecido e foram submetidos a uma radiografia de bacia (anteroposterior) e coxofemoral do lado afetado (perfil). Os pacientes foram operados por abordagem anterolateral ou posterolateral de acordo com a decisão do cirurgião principal e pela mesma equipe de Cirurgia do Quadril de nosso hospital.

Quanto à concepção, o componente femoral da prótese Biomec é de titânio (T16Al7Nb), com ângulo cervicodiafisário de $145^{\circ}$ e formato cuneiforme nos três planos e fixados ao osso sob pressão (pressfit) na região metafisária. A haste proximalmente tem estrias anteriores e posteriores para evitar movimentos rotacionais e favorecer a osteointegração. O acetábulo da Biomec é uma cúpula de titânio, com formato semiesférico, com seis aletas expansíveis. A cabeça intercambiável utilizada foi de cromo-cobaltomobilidênio (CoCrMO) de 28mm.

Para avaliação clínica foram utilizados os critérios propostos por D’Aubigné e Postel ${ }^{(9)}$, que analisa a dor, a marcha e a mobilidade do quadril, com pontuação máxima de seis para cada item, e somatório de 18 pontos para o melhor resultado. Para fim de análise estatística, foram considerados como bons resultados os pacientes que obtiveram graduação entre boa e muito boa (maior ou igual a 15 pontos), e os demais considerados resultados insatisfatórios.

A avaliação radiográfica das osteólises acetabulares baseou-se nos critérios de Zicat et al $^{(10)}$, para o tipo expansivo ou linear e nas zonas de De Lee e Chanrley ${ }^{(11)}$ para sua localização. Na avaliação da osteólise femoral foram usados os critérios de Turíbio ${ }^{(12)}$.

Na avaliação radiográfica também foram avaliadas outras variáveis como o desgaste do polietileno, a migração do componente femoral (subsidence), o índice morfocortical femoral (IMC), todos estes sendo aferidos com régua plástica milimetrada transparente.

Para descrição das variáveis levamos em consideração a média aritmética, o desvio padrão (DP), mediana e os valores máximos e mínimos. Na análise estatística para variáveis não paramétricas foi utilizado o teste de correlação de Spearman e para variáveis paramétricas o teste de Mann-Whitney, ambos calculados pelo programa SPSS for Windows, versão 14.0 (SPSS Inc, Chicago, IL). Consideramos como significativo um valor de P menor que 0,05.

Para verificação de correlação estatística foram avaliados os seguintes desfechos: osteólise acetabular versus desfecho clínico, desfecho clínico versus seguimento, migração femoral e desgaste do polietileno, osteólise femoral e acetabular, osteólise acetabular versus seguimento, desgaste do polietileno e seguimento.

\section{RESULTADOS}

A prótese Biomec foi utilizada em 100 pacientes entre os anos de 1988 e 2001. Destes, sete evoluíram a óbito no pós-operatório tardio por causa não relacionada ao procedimento cirúrgico e, por não apresentarem avaliação recente, foram excluídos do estudo. Outros nove pacientes não foram localizados para reavaliação. Portanto, foram avaliados 84 pacientes e 93 quadris, sendo nove casos com artroplastia bilateral. Dos pacientes operados, 32 eram do sexo masculino (38\%) e 52, do feminino (62\%). A idade média no pré-operatório foi de 47 anos (19 a 77 anos). O lado direito foi operado em 49 casos (58\%) e o esquerdo, em 35 (42\%).

O seguimento médio foi de 10 anos e seis meses, sendo o menor acompanhamento de quatro anos e o maior de 20 anos, com mediana de nove e DP de 4,8 anos.

Ocorreram três casos de falhas de ambos os componentes $(3,2 \%)$ e quatro casos de falha somente do componente acetabular (4,3\%).

Na análise dos resultados clínicos, segundo a classificação de D’Aubigné e Postel ${ }^{(9)}$, obtivemos 82 quadris (88\%) de bons e muito bons resultados, conforme ilustrado na Figura 1. 


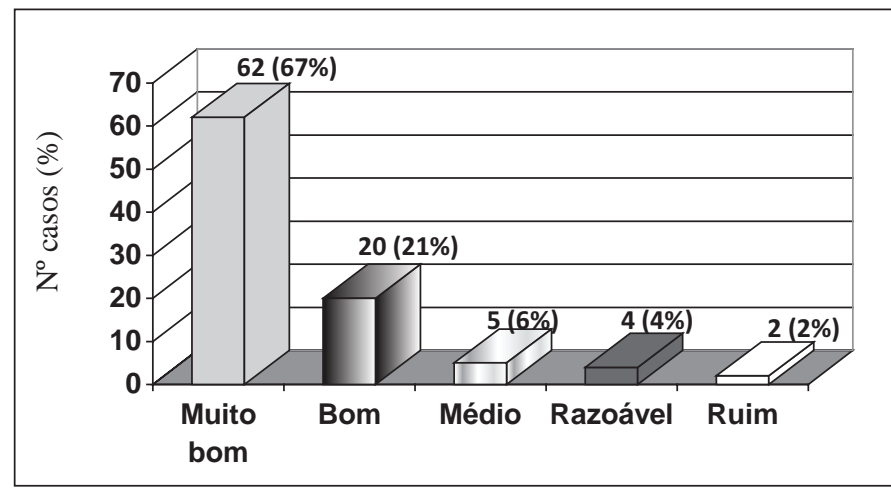

Figura 1 - Distribuição da amostra segundo avaliação funcional proposta por D' Aubigné e Postel ${ }^{(9)}$

As osteólises estiveram presentes em 66 acetábulos (75\%), sendo mais frequentes nas zonas dois e três de DeLee e Chanrley ${ }^{(11)}$, conforme apresentadas na Figura 2, segundo sua localização.

Na Figura 3 apresentamos uma radiografia de um caso com 17 anos de seguimento do lado direito e 16

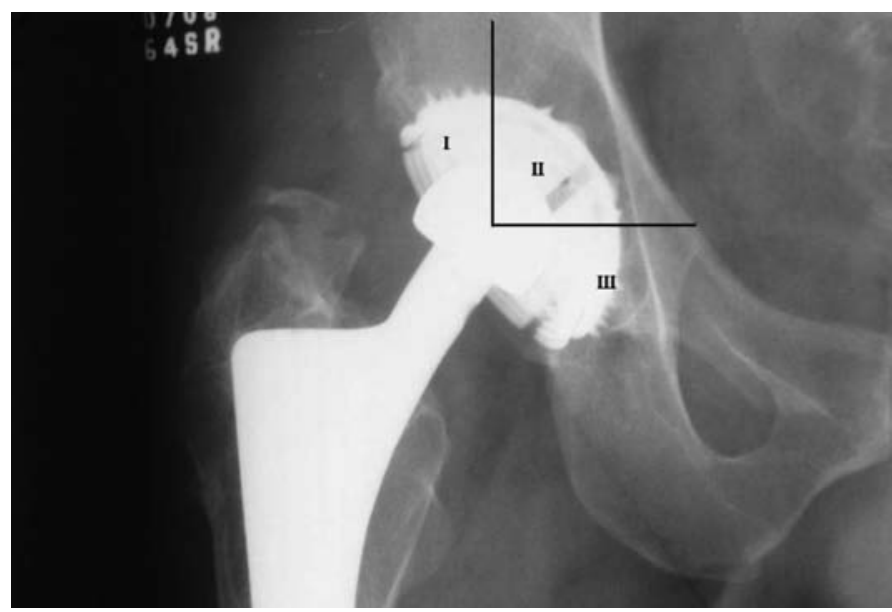

Figura 2 - Distribuição da amostra segundo presença de osteólise e localização conforme áreas de DeLee e Chanrley ${ }^{(11)}$. Neste caso consideramos osteopenia na área II

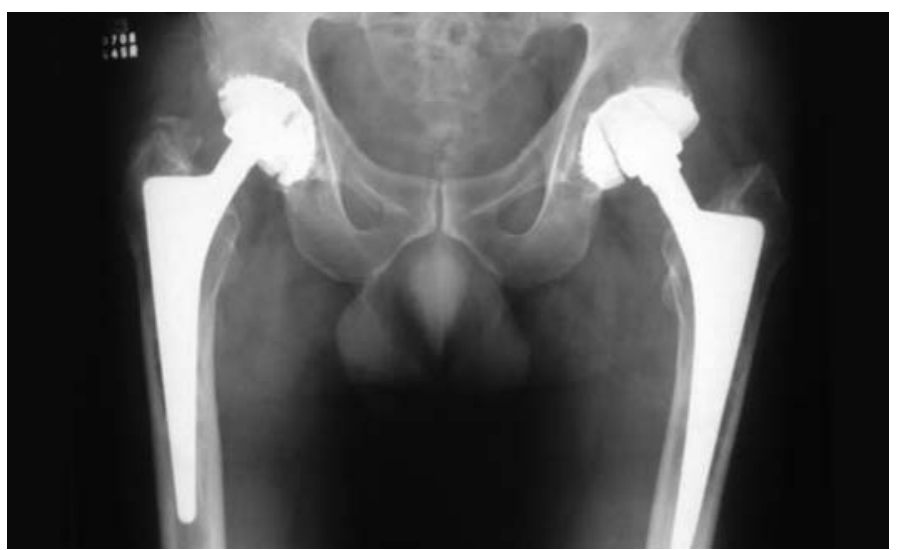

Figura 3 - Radiografia AP bacia de um paciente com 17 anos de seguimento à direita e 16 anos à esquerda anos do lado esquerdo, sem sinais de afrouxamento dos implantes e com sinais de osteólise acetabular, mas sem repercussão clínica.

As osteólises femorais estiveram presentes em 83 casos (98,8\%), e foram classificadas segundo critérios de Turíbio conforme ilustrado na Tabela 1 de acordo com a sua localização:

Tabela 1 - Distribuição da amostra em relação à osteólise femoral conforme critérios de Turíbio

\begin{tabular}{c|c|c}
\hline $\begin{array}{c}\text { Graduação de Turíbio } \\
\text { (Grau) }\end{array}$ & Casos & Porcentagem (\%) \\
\hline 0 & 1 & 1,20 \\
1 & 29 & 34,50 \\
2 & 42 & 50,00 \\
3 & 10 & 11,90 \\
4 & 2 & 2,40 \\
\hline
\end{tabular}

0 - ausência de osteólise

1 - osteólise restrita à região do grande trocanter

2 - osteólise estendendo-se da região do grande trocanter em direção ao pequeno trocanter

3 - osteólise acometendo o grande e o pequeno trocanter estendendo-se até o terço médio diafisário 4 - osteólise em toda a regiâo do implante femoral.

Na Figura 4 apresentamos um exemplo de osteólise femoral acometendo a região do grande e pequeno trocanter (Turíbio 2), sem comprometer a estabilidade do componente femoral.

O desgaste do polietileno esteve presente em 69 casos (82\%), com média de 1,4mm ( 0 a 5). Se considerarmos o seguimento médio de 10 anos e seis meses, o

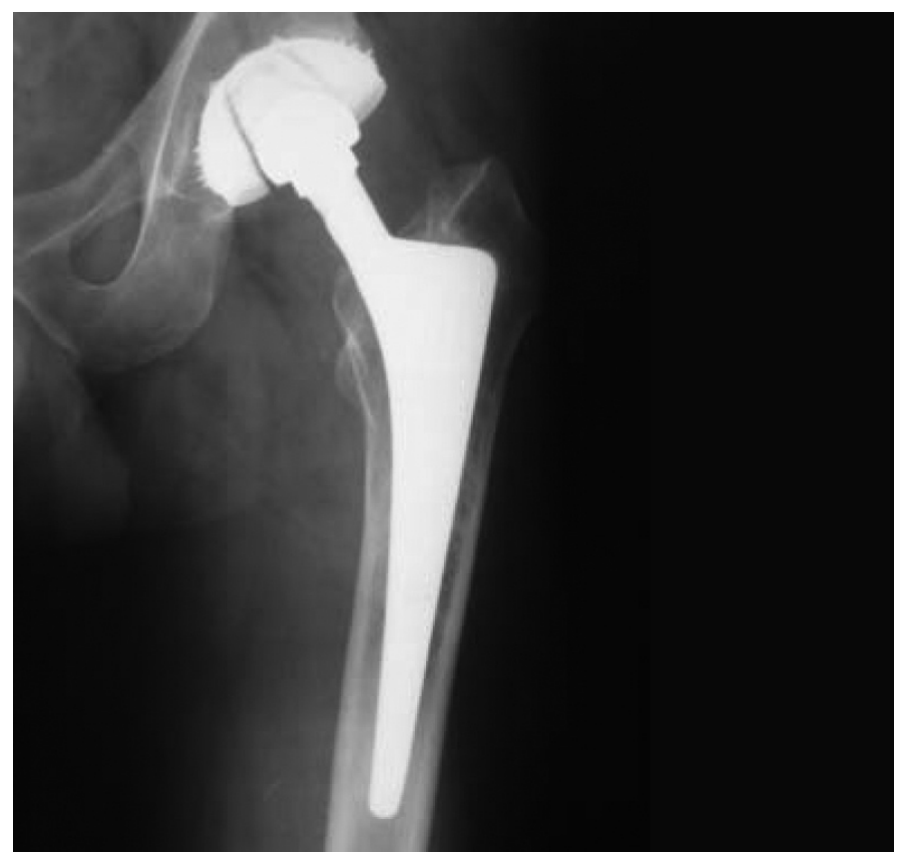

Figura 4 - Radiografia de quadril esquerdo demonstrando osteopenia femoral acometendo região do grande e do pequeno trocanter sem comprometimento da estabilidade e fixação do implante femoral 
desgaste médio do polietileno foi de $0,13 \mathrm{~mm}$ por ano. A análise estatística demonstrou que a osteólise acetabular esteve relacionada ao desgaste do polietileno $(\mathrm{P}=0,04)$. $\mathrm{O}$ desgaste do polietileno também esteve correlacionado com uma piora no desfecho clínico $(\mathrm{P}=0,036)$.

A presença de migração da haste femoral ou "subsidence" esteve presente em 67 casos (79,7\%), ausente em 17 (20,3\%), com uma média de migração de 2,33mm e mediana de $2 \mathrm{~mm}$. A Figura 5 ilustra melhor a frequência das migrações:

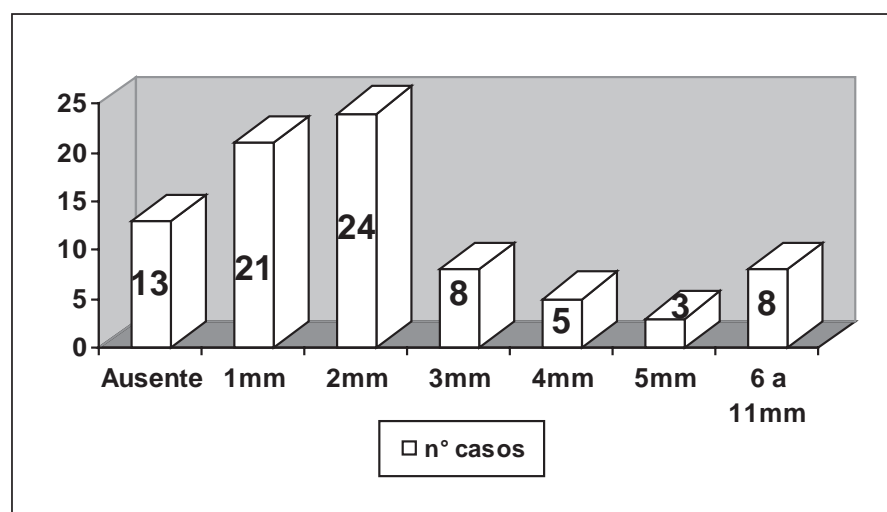

Figura 5 - Distribuição da amostra de acordo com a frequência e magnitude da migração da haste femoral

A análise estatística não mostrou piora do desfecho clínico com a migração femoral $(\mathrm{P}=0,009)$.

\section{DISCUSSÃO}

A distribuição de nossa amostra em relação ao sexo (38\% homens e 62\% de mulheres) esteve de acordo com os artigos revisados ${ }^{(13-15)}$.

A média de idade encontrada em nosso trabalho de 47 anos foi semelhante aos trabalhos encontrados, que relatam média de idade de 51 a 53 anos $^{(13-18)}$.

A avaliação clínica com $80 \%$ de resultados bons e muito bons também foi similar à encontrada em estudo com a prótese de Spotorno ${ }^{\circledR}$, modelo estrangeiro em que foi baseado o desenvolvimento da prótese em estudo $^{(13,14,18,19)}$. Na análise estatística do desfecho clínico com o seguimento observou-se uma diminuição da graduação funcional com o aumento do seguimento, com um $\mathrm{P}=0,008$; o que se justifica que com o passar dos anos e desgaste do implante ocorra uma diminuição gradual do escore clínico.

Não encontramos significância estatística quando analisamos a prevalência de osteólise acetabular (75\%) com o desfecho clínico $(\mathrm{P}=0,001)$. Este dado justifica o achado de resultados funcionais muito bons apesar da alta prevalência de osteólise acetabular. Este dado vai ao encontro do que foi relatado por outros autores em que não encontraram relação da osteólise acetabular com o desfecho clínico ${ }^{(15,18)}$.

A osteólise femoral apresentou uma prevalência ainda maior, sendo observada em $98,8 \%$ dos casos. No entanto, aproximadamente $85 \%$ delas acometeram somente a região do grande e pequeno trocanter, não comprometendo a fixação e/ou a estabilidade do implante. Este dado é demonstrado em estudo prospectivo com avaliação da densidade mineral óssea periprótese após artroplastia de Spotorno ${ }^{\circledR}$, em que se encontrou um declínio importante da massa óssea (21\%) na região trocantérica nos primeiros dois anos pós-operatórios ${ }^{(20)}$. $\mathrm{Na}$ análise estatística da osteólise femoral com o desfecho clínico também não encontramos significância estatística $(\mathrm{P}=0,124)$.

A osteólise acetabular apresentou correlação com a femoral, apresentando uma prevalência maior quanto mais acentuada a osteólise femoral $(\mathrm{P}=0,014)$. Isto se deve muito provavelmente pelo acúmulo de debris e penetração na interface osso-implante.

A correlação do desgaste do polietileno com uma maior prevalência de osteólise acetabular encontrada em nosso trabalho foi estatisticamente significativa $(\mathrm{P}=0,04)$ e já havia sido demonstrada por trabalhos anteriores ${ }^{(21,22)}$.

O desgaste médio de 1,4mm encontrado na série e o desgaste anual considerando o seguimento médio de 10,6 anos de $0,13 \mathrm{~mm} /$ ano podem ser sobrepostos ao desgaste médio encontrado na literatura para próteses cimentadas, e demonstrou de menor monta quando comparado com trabalhos de acetábulos não cimentados (não expansivos) com seguimento menor ${ }^{(23)}$.

A migração e acomodação da haste femoral (subsidence) em 67 casos (80\%), com média de 2,33mm, vêm ao encontro da teoria biomecânica há tempo defendida por Spotorno para hastes cuneiformes não cimentadas, e atualmente bastante valorizadas pelos defensores das hastes cuneiformes polidas cimentadas e sem colar ${ }^{(24)}$. Estes dados nos fazem crer que até estabelecer-se a fixação secundária pela osteointegração, possa e deva ocorrer o ajustamento da haste quadrangular cuneiforme dentro do estojo ósseo.

\section{CONCLUSÃO}

A ATQ com a prótese não cimentada Biomec apresentou $88,2 \%$ de bons resultados em 10 anos e seis meses de seguimento médio nessa coorte.

Apesar de encontrarmos osteólises femorais e acetabulares em número significativo em nossa amostra, elas não tiveram significância na alteração do desfecho clínico. 


\section{REFERÊNCIAS}

1. Charnley J. The long-term results of low-friction arthroplasty of the hip performed as a primary intervention. J Bone Joint Surg Br. 1972;54(1):61-76.

2. Jones LC, Hungerford DS. Cement disease. Clin Orthop Relat Res. 1987;(225):192-206.

3. Chandler HP, Reineck FT, Wixson RL, McCarthy JC. Total hip replacement in patients younger than thirty years old. A five-year follow-up study. J Bone Joint Surg Am. 1981;63(9):1426-34.

4. Judet R, Siguier M, Brumpt B, Judet T. A noncemented total hip prosthesis. Clin Orthop Relat Res. 1978;(137):76-84.

5. Spotorno L, Romagnoli S, Ivaldo N, Grappiolo G, Bibbiani E, Blaha DJ, Guen TA.The CLS system. Theoretical concept and results. Acta Orthop Belg. 1993;59(Suppl 1):144-8.

6. Macedo CAS, Scheidt RB, Palma HM, Rosito R, Weissheimer W, Galia CR. Artroplastia total de quadril de spotorno: resultados clínicos e radiográficos. Acta Ortop Bras. 2008;16(1):17-22.

7. Zweymüller K, Semlitsch M. Concept and material properties of a cementless hip prosthesis system with $\mathrm{Al} 2 \mathrm{O} 3$ ceramic ball heads and wrought Ti-6Al-4V stems. Arch Orthop Trauma Surg. 1982;100(4):229-36.

8. Zweymüller K. A cementless titanium hip endoprosthesis system based on press-fit fixation: basic research and clinical results. Instr Course Lect. 1986;35:203-25.

9. D'Aubigné RM, Postel M. Function al results of hip arthroplasty with acrylic prosthesis. J Bone Joint Surg Am. 1954;36(3):451-75.

10. Zicat B, Engh CA, Gokcen E. Patterns of osteolysis around total hip components inserted with and without cement. J Bone Joint Surg Am. 1995;77(3):432-9.

11. DeLee JG, Charnley J. Radiological demarcation of cemented sockets in total hip replacement. Clin Orthop Relat Res. 1976;(121):20-32.

12. Turíbio FM. Alterações radiográficas do fêmur nas artroplastias totais do quadril com prótese não cimentada de Parhofer-Monch [tese]. São Paulo: Escola Paulista de Medicina, Universidade Federal de São Paulo; 1996.

13. Spotorno L, Schenk RK, Dietschi C, Romagnoli S, Mumenthaler A. [Personal experiences with uncemented prostheses]. Orthopade. 1987;16(3):225-38.
14. Bulow JU, Scheller G, Arnold P, Synatschke M, Jani L. Follow-up (6-9 years) results of the uncemented CLS Spotorno stem. Arch Orthop Trauma Surg. 1996;115(3-4):190-4.

15. Aldinger PR, Breusch SJ, Lukoschek M, Mau H, Ewerbeck V, Thomsen M. A ten- to 15-year follow-up of the cementless spotorno stem. J Bone Joint Surg Br. 2003;85(2):209-14.

16. Schramm M, Keck F, Hohmann D, Pitto RP. Total hip arthroplasty using an uncemented femoral component with taper design: outcome at 10-year follow-up. Arch Orthop Trauma Surg. 2000;120(7-8):407-12.

17. Vidyadhara S, Rao SK. Uncemented primary press-fit total hip arthroplasty: a 3 to 6 years of experience. J Orthop Surg (Hong Kong). 2007;15(1):50-5.

18. Tauber C, Kidron A. Total hip arthroplasty revision using the press-fit CLS Spotorno cementless stem. Twenty-four hips followed between 1987 and 1998. Arch Orthop Trauma Surg. 2000;120(3-4):209-11.

19. Macedo CAS, Scheidt RB, Palma HM, Rosito R, Weissheimer W, Galia CR. Artroplastia total do quadril de Spotorno. Acta Ortop Bras. 2008;16(4):217-22.

20. Zerahn B, Lausten GS, Kanstrup IL. Prospective comparison of differences in bone mineral density adjacent to two biomechanically different types of cementless femoral stems. Int Orthop. 2004;28(3):146-50.

21. Mallory TH, Head WC, Lombardi AV Jr. Tapered design for the cementless total hip arthroplasty femoral component. Clin Orthop Relat Res. 1997;(344):172-8.

22. Robinson RP, Deysine GR, Green TM. Uncemented total hip arthroplasty using the CLS stem: a titanium alloy implant with a corundum blast finish. Results at a mean 6 years in a prospective study. J Arthroplasty. 1996;11(3):286-92.

23. Joshi RP, Eftekhar NS, McMahon DJ, Nercessian OA. Osteolysis after Charnley primary low-friction arthroplasty. A comparison of two matched paired groups. J Bone Joint Surg Br. 1998;80(4):585-90.

24. Engh CA, Massin P, Suthers KE. Roentgenographic assessment of the biologic fixation of porous-surfaced femoral components. Clin Orthop Relat Res. 1990;(257):107-28. 